\title{
Hand-made Extracorporeal Knotting versus Hem-o-Lok Clip for Stump Closure in Laparoscopic Appendectomy
}

\author{
Ilker Murat Arer, Tevfik Avci and Hakan Yabanoglu \\ Department of General Surgery, Baskent University Adana Teaching and Research Center, Adana, Turkey
}

\begin{abstract}
Objective: To compare hand-made extracorporeal knotting and hem-o-lok clip for the closure of appendix stump regarding safety, complications and cost-effectiveness.

Study Design: Descriptive study.

Place and Duration of Study: Baskent University Adana Teaching and Research Center, between January 2010 and December 2016.

Methodology: A total of 287 patients, who underwent laparoscopic appendectomy, were enrolled in this study. Patients were divided into hand-made extracorporeal knotting and hem-o-lok clip groups. Patients were compared according to age, gender, duration of symptoms, serum leukocyte count, American Society of Anesthesiologists (ASA) score, intraoperative findings, operation time, hospital stay, pathology report and complications.

Results: Of 287 patients, $149(51.9 \%)$ were female and $138(48.1 \%)$ were male. The mean age was found 34.9 years. No statistical difference was observed between groups according to age, gender, duration of symptoms, serum leukocyte count, ASA score, operation time and hospital stay. Intraoperative findings were different between groups $(p<0.05)$. Overall postoperative complication rate was $6.6 \%$. No statistical difference was observed regarding complications

Conclusion: Both hand-made extracorporeal knotting and hem-o-lok clips can be used for stump closure in laparoscopic appendectomy. Hand-made extracorporeal knotting is an effective, safe and costeffective method alternative to hem-o-lok clips.
\end{abstract}

Key Words: Laparoscopic appendectomy, Clip, Stump closure, Knotting, Appendicitis.

How to cite this article: Arer IM, Avci T, Yabanoglu H. Hand-made extracorporeal knotting versus hem-o-lok clip for stump closure in laparoscopic appendectomy. J Coll Physicians Surg Pak 2019; 29(12):1203-1206.

\section{INTRODUCTION}

Appendectomy is the most commonly performed surgical procedure for patients admitted to emergency department. Since laparoscopic appendectomy (LA) was first introduced in 1983, it became popular and an alternative to open appendectomy. ${ }^{1}$ LA is now the standard approach for the treatment of acute appendicitis and superior to open appendectomy regarding outcomes. It has the advantage of better visualisation of the abdominal cavity for additional pathologies. However, LA has higher cost rates than open appendectomy because of stump closure technique.

There are different techniques for appendix stump closure in laparoscopic surgery. ${ }^{2}$ Some authors recommend simple tie knotting or endoloop3; whereas, others recommend staplers ${ }^{4}$ and clips. ${ }^{5}$ All these techniques were proven to be safe and effective but stapler and endoloop increase the cost, especially stapler should be preferred only in selected patients. 6 Metal clips were

Correspondence to: Ilker Murat Arer, Department of General Surgery, Baskent University Adana Teaching and Research

Center, Dadaloglu District 2591 Street No:4/A 01250 Yuregir, Adana, Turkey

E-mail: igy1981@yahoo.com

Received: January 10, 2019; Revised: May 19, 2019; Accepted: June 19, 2019 found to be effective and safe even in complicated appendicitis. ${ }^{7}$ Simple tie mimics endoloop but decreases cost; and it can be applied by intra- or extra-corporeally. Recently, hem-o-lok (non-absorbable polymeric) clips were introduced and found to be effective for stump closure. ${ }^{8}$ Hand-made extracorporeal knotting was described before 9 but it was not compared with endoclip.

As described above, all techniques for stump closure can be preferred; however, their cost-effectiveness and applicability should be considered. There are fewer studies in literature comparing hand-made extracorporeal knotting and hem-o-lok clips. In this study, the aim was to compare two different techniques; handmade extracorporeal knotting and hem-o-lok clip for stump closure in laparoscopic appendectomy regarding operation time, complications and cost-effectiveness; and define which one safer and superior.

\section{METHODOLOGY}

A total of 287 patients who underwent laparoscopic appendectomy at Baskent University between January 2010 and December 2016 were included. Patients with the signs of clinical and/or radiological appendicitis, age between 18-90 years, laparoscopic appendectomy with use of hand-made extracorporeal knotting and hem-o-lok clip were included. Patients with plastrone appendicitis, conversion to open appendectomy, single 
incision laparoscopic appendectomy and use of other techniques for stump closure were excluded. Data were collected retrospectively via patient software data records. Patients were divided into two groups according to stump closure technique: group 1 was hem-o-lok clip and group 2 was hand-made extracorporeal knotting group. Ethical approval and informed consent were not taken beacuse of the retrospective design of this study, but this study was prepared in accordance with the Declaration of Helsinki ethical principles.

All operations were perfromed using 3 conventional laparoscopic trocars (two 10-mm and one 5-mm ports). One 10-mm trocar for the camera (30 degree) was placed via infraumbilical incision, other 10-mm trocar via suprapubic incision and 5-mm trocar via right lower quadrant mid-clavicular incision. After positioning the patient in $20^{\circ}$ Trendelenburg position and $15^{\circ}$ tilting to the left, dissection of the mesoappendix was done gently by Ultracision ${ }^{\circledR}$ harmonic scalpel (Ethicon Endosurgery, Cincinnati, USA). Then appendix stump was closed by modified Tayside knot, hand-made extracorporeal knotting described by Mayir et al. ${ }^{10}$ Stump was closed by two knots in group 2 (Figure 1). One non-absorbable polyester braided suture or absorbable polyglactin braided suture was tied outside the abdomen and then pushed forward by the knot applier on the appendix stump. Nearly 2-3 mm above the former knot, another one was placed. Non-absorbable polymeric clip (Hem-olok ${ }^{\circledR}$ ligation system, Teleflex Medical, NC, (USA) was used for patients in group 1. One clip was placed on the appendix stump by clip applier. The other clip was placed 2-3 $\mathrm{mm}$ above the former one (Figure 2).

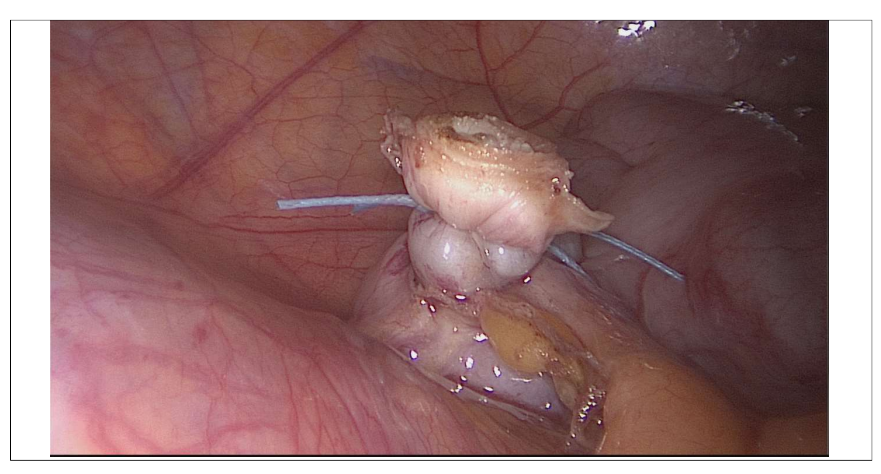

Figure 1: Hand-made extracorporeal knotting on appendix stump.

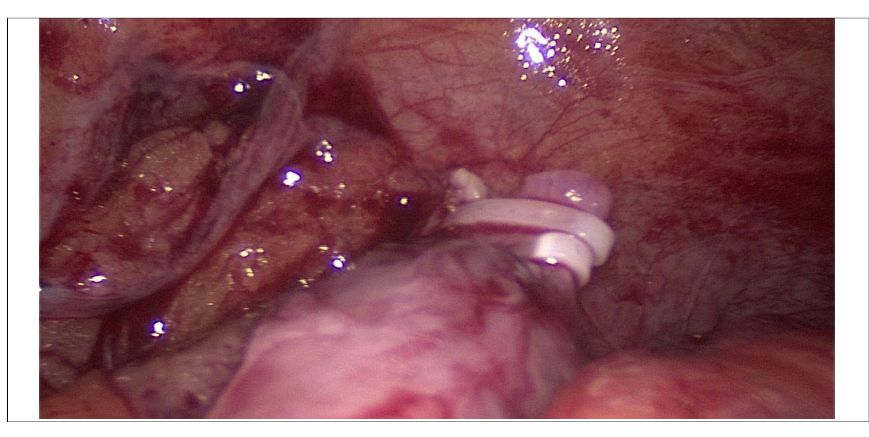

Figure 2: Hem-o-lok clips on appendix stump.
Patients in the two groups were compared according to age, gender, duration of symptoms, serum leukocyte count, American Society of Anesthesiologists (ASA) score, intraoperative findings, operation time, hospital stay, pathology report and complications.

Statistical analyses were performed with Statistical Package for the Social Sciences Software (version 17.0; SPSS, Inc., Chicago, IL). For continuous variables, Kolmogorov-Smirnov test was applied to test normal distribution. The two groups were compared using the independent samples t-test for normally distributed continuous variables and the Mann-Whitney U-test for non-normally distributed continuous variables. For continuous variables, those with normal distribution were expressed as mean $\pm S D$, median values without normal distribution. The Chi-square test was used for compassion of categorical variables. A p-value less than 0.05 was considered statistically significant.

\section{RESULTS}

Of 287 patients, $149(51.9 \%)$ were females and 138 $(48.1 \%)$ were males. The mean age was found 34.9 years (min. 18 max. 79 years). Demographic data of the patients were described on Table I. One hundred and eight patients were in group 1 and 179 patients in group 2. Mean duration of symptoms was 1.79 and 1.73 days for group 1 and 2, respectively. Average serum leukocyte count of patients in two groups were similar $(p=0.790)$. There was no statistical difference between groups regarding ASA score $(p=0.081)$. Perforated appendicitis was found intraoperatively in $11(6.1 \%)$ patients that were in group 2 and intraabdominal abscess was found in $2(1.1 \%)$ patients that were also in group 2 . None of the patients in group 1 were found to have perforated appendicitis or abscess intraoperatively, which was statistically different $(p=0.016)$. The average operation time was found to be 55 and 45 minutes for group 1 and 2 , respectively (Table II). The average hospital stay for group 1 was 1 day and for group 2 was 1 day $(p=0.038)$. The permanent pathology of $12(11.1 \%)$ patients in group 1 and $8(4.5 \%)$ patients in group 2 were reported as perforated appendicitis $(p=0.053)$. Superficial incisional surgical site infection $(4.5 \%)$ was the most common complication which was observed in 13 patients. Overall postoperative complication rate was $6.6 \%(n=19)$. No patients received surgical re-intervention. Percutaneous intraabdominal abscess drainage was done in $4(1.4 \%)$ patients. No intra-operative complication and postoperative mortality was observed.

Two hem-o-lok clips used for each patient in group 1 , accounts for $\$ 20$ and 1 suture used for each patient in group 2 accounts for $\$ 0.63$, except from the operation cost. Regarding cost-effectiveness, hem-o-lok clip cost is 31.7 times suture material.

\section{DISCUSSION}

Laparoscopic appendectomy is gaining popularity than open procedure and has the advantages such as shorter 
Table I: Demographic data of patients.

\begin{tabular}{|c|c|c|c|}
\hline & $\begin{array}{c}\text { Hem-o-lok } \\
\text { group } \\
(n=108)\end{array}$ & $\begin{array}{c}\text { Hand-made } \\
\text { extracorporeal } \\
\text { knotting group } \\
(n=179)\end{array}$ & $p$-value \\
\hline Female/Male & $55 / 53$ & $94 / 85$ & $0.808\left(\chi^{2}=0,068\right)$ \\
\hline $\mathrm{Age}^{*}$ (years), median (IQR) & $31.5(18-79)$ & $32(18-78)$ & $0.522(Z=-0.64)$ \\
\hline $\begin{array}{l}\text { Duration of symptoms* } \\
\text { (Days), median (IQR) }\end{array}$ & $1(1-10)$ & $1(1-10)$ & $0.858(Z=-0.179)$ \\
\hline $\begin{array}{l}\text { Serum leukocyte count } \\
(\times 103 / \mu \mathrm{L})\end{array}$ & $13.63 \pm 5.16$ & $13.48 \pm 3.88$ & $0.790(\mathrm{t}=0.267)$ \\
\hline ASA score & & & $0.081\left(\chi^{2}=5.028\right)$ \\
\hline 1 & $76(70.4 \%)$ & $106(59.2 \%)$ & \\
\hline 2 & $25(23.1 \%)$ & $64(35.8 \%)$ & \\
\hline 3 & $7(6.5 \%)$ & $9(5 \%)$ & \\
\hline Intraoperative finding & & & $0.016\left(\chi^{2}=8.216\right)$ \\
\hline Appendicitis & $108(100 \%)$ & $166(92.7 \%)$ & \\
\hline Perf. appendicitis & $0(0 \%)$ & $11(6.1 \%)$ & \\
\hline Abscess & $00 \%)$ & $2(1.1 \%)$ & \\
\hline Pathology report & & & $0.053\left(\chi^{2}=4.583\right)$ \\
\hline Appendicitis & $96(88.9 \%)$ & $171(95.5 \%)$ & \\
\hline Perf. appendicitis & $12(11.1 \%)$ & $8(4.5 \%)$ & \\
\hline
\end{tabular}

* Values are mean \pm standard deviation.

ASA $=$ American society of anesthesiologists.

Table II: Per- and post-operative findings of the patients.

\begin{tabular}{l|c|c|c}
\hline & $\begin{array}{c}\text { Hem-o-lok } \\
\text { group } \\
(\mathrm{n}=108)\end{array}$ & $\begin{array}{c}\text { Hand-made } \\
\text { extracorporeal } \\
\text { knotting group } \\
(\mathrm{n}=179)\end{array}$ & $\mathrm{p}$-value \\
\hline $\begin{array}{l}\text { Operation time (minutes), } \\
\text { median (IQR) }\end{array}$ & $55(15-120)$ & $45(20-120)$ & $0.317(\mathrm{Z}=1)$ \\
\hline Hospital stay (days), & $1(1-12)$ & $1(1-8)$ & $0.038(\mathrm{Z}=2.075)$ \\
median (IQR) & $102(94.4 \%)$ & $166(92.7 \%)$ & $0.111\left(\chi^{2}=6.010\right)$ \\
\hline Complication & $4(3.7 \%)$ & $9(5 \%)$ & \\
$\quad$ None & $0(0 \%)$ & $4(2.2 \%)$ & \\
$\quad$ SISSI & $2(1.9 \%)$ & $0(0 \%)$ & \\
Intraabdominal abscess & \multicolumn{2}{l}{ Seroma } &
\end{tabular}

SISSI: Superficial incisional surgical site infection.

hospital stay, fast recovery, less pain, and better quality of life. Stump closure of the appendix is the crucial step of this procedure. Although some authors use only coagulation devices for stump closure, most of the surgeons do not dare that much yet. Stump leakage may result in complications such as intraabdominal abscess. In open appendectomy stump can be easily secured with simple tie or in complicated cases with purse string suture. For safe closure of the stump in LA, there are several techniques that are: intra- or extra-corporeal simple tie, endoloop, metal or polymeric clips and endostaplers. ${ }^{7}$ There are studies comparing these techniques although comparison of hand-made extracorporeal knotting and polymeric clips has not been done yet. Strzalka et al. conducted a study comparing metal clip, suture and endostaplers in 307 laparoscopic appendectomy patients; and found no statistical difference regarding complications between these three techniques. ${ }^{11}$ Matyja et al. also compared these three methods, found no differences between these techniques, and states that endoscopic stapler increases cost and should be applied in cases of failure of stump closure with other techniques. ${ }^{12}$

Both of these studies are retrospective, which is also one of the limitations of our study. However, endostaplers are proven to be safe even in complicated cases, they increase the cost a lot. ${ }^{13,14}$ Application of the endostapler may increase operation time. Some authors observed increased operation time in endostapler group; 6 whereas, Kazemier et al. found the opposite.13 Together with increasing the cost and operation time, we believe that the application of endostapler via $15 \mathrm{~mm}$ trocar also increases risk of trocar site hernia. Therefore, endostapler should be used in cases when other techniques fail to secure the appendix stump.

Endoloop and intracorporeal knotting have been compared by Bali et al. and no statistical difference between groups other than operation time was observed. ${ }^{15}$ The mean operation time increases nearly 5.6 minutes when intracorporeal knotting was applied $(p=0.01)$. Kiudelis et al. conducted a prospective study on 152 patients comparing intracorporeal knotting with invaginating suture versus endoloop and found that endoloop decreases operation time $(p>0.05)$, but increases cost approximately $80 €$ for each patinet. ${ }^{16}$ Therefore, endoloop has the advantage of less operation time but increase in cost.

Hem-o-lok clips made of non-absorbable polymeric material can also be used for stump closure. Hue et al. compared hem-o-lok clips and endoloop in 105 patients and found hem-o-lok clips to be safe, fast and costeffective in patients with a mildly to moderately inflamed appendix base of less than $10 \mathrm{~mm}$ in diameter. $17 \mathrm{Hem}$ o-lok clips are $\$ 39$ cheaper than endoloop. ${ }^{17}$ They are also easily applied and do not increase operation time. In our opinion, hem-o-lok clips are safer than metal clips because they have locking mechanism. Rickert et al. used titanium double shanked clips for stump closure, which have the similar design with hem-o-lok clips and found it to be safe and cost-effective. 18 Unfortunately, these titanium clips are applied via $12.5 \mathrm{~mm}$ trocar; whereas, hem-o-lok clips via $11 \mathrm{~mm}$ trocar. Ates et al. compared titanium endoclip and intracorporeal knot tying in a prospective randomised study, including 61 patients, and found titaniun endoclips to be a useful alternative to intracorporeal knot-tying and titanium endoclip application significantly decreases operation time $(p=0.01) .{ }^{15}$

In a study including 49 pediatric patients, hem-o-lok clips were found to be easily applicable (the mean duration of application for hem-o-lok is 1 minute and endoloop is 5 minutes, $p<0.05$ ) and more economical (by \$81.5) than endoloops. ${ }^{19}$ Colak et al. also found hem-o-lok group to have shorter operation time and cheaper than endoloop group. 20 Lucchi et al. stated that endoloop and hem-o-lok clips are safe for appendicular stump closure although hem-o-lok clips are superior in case of ease- 
ness of use and cheapness. ${ }^{21}$ Wilson et al. found hemo-lok clips to be safe as endoloop with the advantages of low cost and less operation time. 22 However, there are cheaper endoloops that costs between \$15-32 and equal to hem-o-lok clip cost. 6

Mayir et al. described hand-made loop for stump closure which is an effective, safe and cheap (only \$1) method. ${ }^{10}$ Although hem-o-lok clip application makes additional cost $\$ 19.37$ to the patient, it is also inexpensive compared to other techniques. ${ }^{23}$ Hand-made loops do not increase operation time and can even be applied via 5-mm trocar.

\section{CONCLUSION}

Hand-made loop and hem-o-lok clips are both used for stump closure in laparoscopic appendectomy. But, hand-made loop is an effective, safe and cost-effective alternative method to hem-o-lok clips.

\section{CONFLICT OF INTEREST:}

Authors declared no conflict of interest.

\section{AUTHORS' CONTRIBUTION:}

IMA: Conception or design of the work, analysis, interpretation of data, final approval of the version to be published, critical review.

TA: Interpretation of data, drafting the work, final approval of the version to be published.

HY: Conception or design of the work, conception or design of the work, drafting the work, final approval of the version to be published, critical review.

\section{REFERENCES}

1. Semm K. Endoscopic appendectomy. Endoscopy 1983; 15:59-64.

2. Delibegovic $S$, Mehmedovic $Z$. The influence of the different forms of appendix base closure on patient outcome in laparoscopic appendectomy: a randomized trial. Surg Endosc 2018; 32:2295-9.

3. Sahm M, Kube R, Schmidt S, Ritter C, Pross M, Lippert H. Current analysis of endoloops in appendiceal stump closure. Surg Endosc 2011; 25:124-29.

4. Beldi G, Vorburger SA, Bruegger LE, Kocher T, Inderbitzin D, Candinas D. Analysis of stapling versus endoloops in appendiceal stump closure. Br J Surg 2006; 93:1390-3.

5. Ates M, Dirican A, Ince V, Ara C, Isik B, Yilmaz S. Comparison of intracorporeal knot-tying suture (polyglactin) and titanium endoclips in laparoscopic appendiceal stump closure: A prospective randomized study. Surg Laparosc Endosc Percutan Tech 2012; 22:226-31.

6. Rakic M, Jukic M, Pogorelic Z, Mrklie I, Klicek R, Družijanic N, et al. Analysis of endoloops and endostaples for closing the appendiceal stump during laparoscopic appendectomy. Surg Today 2014; 44:1716-22.

7. Gomes CA, Junior CS, de Peixoto RO, Netto JM, Gomes CC, Gomes FC. Appendiceal stump closure by metal endoclip in the management of complicated acute appendicitis. World $J$ Emerg Surg 2013; 8:35.

8. Hanssen A, Plotnikov S, Dubois R. Laparoscopic appendec- tomy using a polymeric clip to close the appendicular stump. JSLS 2007; 11:59-62.

9. Kothari R, Somashekar U, Sharma D, Thakur DS, Kumar V. A simple and safe extracorporeal knotting technique. JSLS 2012; 16:280-2.

10. Mayir B, Bilecik T, Ensari CO, Oruc MT. Laparoscopic appendectomy with hand-made loop. Wideochir Inne Tech Maloinwazyjne 2014; 9:152-6.

11. Strzaaka M, Matyja M, Rembiasz K. Comparison of the results of laparoscopic appendectomies with application of different techniques for closure of the appendicular stump. World $\mathrm{J}$ Emerg Surg 2016; 11:4.

12. Matyja M, Strzalka M, Rembiasz K. Laparosocopic appendectomy, cost-effectiveness of three different techniques used to close the appendix stump. Pol Przegl Chir 2015; 87:634-7.

13. Kazemier G, in't Hof KH, Saad S, Bonjer HJ, Sauerland S. Securing the appendiceal stump in laparoscopic appendectomy: Evidence for routine stapling? Surg Endosc 2006; 20:1473-6.

14. Partecke LI, Kessler W, Patrzyk M, Heidecke CD, Bernstorff WV. Comparison among different closure methods of the appendicular stump in laparoscopic appendectomy. Surg Technol Int 2011; 21:85-91.

15. Bali I, Karateke F, Özyazici S, Kuvvetli A, Oruç C, Menekse E, et al. Comparison of intracorporeal knotting and endoloop for stump closure in laparoscopic appendectomy. Ulus Travma Acil Cerrahi Derg 2015; 21:446-9.

16. Kiudelis M, Ignatavicius $P$, Zviniene K, Grizas S. Analysis of intracorporeal knotting with invaginating suture versus endoloops in appendiceal stump closure. Wideochir Inne Tech Maloinwazyjne 2013; 8:69-73.

17. Hue CS, Kim JS, Kim KH, Nam SH, Kim KW. The usefulness and safety of Hem-o-lok clips for the closure of appendicular stump during laparoscopic appendectomy. J Korean Surg Soc 2013; 84:27-32.

18. Rickert A, Bönninghoff R, Post S, Walz M, Runkel N, Kienle P. Appendix stump closure with titanium clips in laparoscopic appendectomy. Langenbecks Arch Surg 2012; 397:327-31.

19. Akbiyik F, Senel E, Bayram-Kabacam G, Demirkan H, Atayurt H, Tiryaki T. A comparison of polymer clips and endoloop applications for securing the appendiceal stump during laparoscopic surgery in children. Surg Laparosc Endosc Percutan Tech 2011; 21:349-52.

20. Colak E, Kement M, Ozlem N, Mutlu T, Yildirim K, Gurer A, et al. A comparison of nonabsorbable polymeric clips and endoloop ligatures for the closure of the appendicular stump in laparoscopic appendectomy: a prospective, randomized study. Surg Laparosc Endosc Percutan Tech 2013; 23:255-8.

21. Lucchi A, Berti P, Grassia M, Siani LM, Gabbianelli C, Garulli G. Laparoscopic appendectomy: Hem-o-lok versus endoloop in stump closure. Updates Surg 2017; 69:61-5.

22. Wilson M, Maniam P, Ibrahim A, Makaram N, Knight SR, Patil P. Polymeric clips are a quicker and cheaper alternative to endoscopic ligatures for securing the appendiceal stump during laparoscopic appendicectomy. Ann R Coll Surg Engl 2018; 100:454-8.

23. Parlakgumus A, Ezer A. Polymeric clips for stump closure in laparoscopic appendectomy. J Coll Physicians Surg Pak 2017; 27:660-2. 